“ (C) 2017 IEEE. Personal use of this material is permitted. Permission from IEEE must be obtained for all other uses, in any current or future media, including

reprinting/republishing this material for advertising or promotional purposes, creating new collective works, for resale or redistribution to servers or lists, or reuse of any copyrighted component of this work in other works." 


\section{Non-Parametric Data Optimization for 2D Laser Based People Tracking}

\author{
Zulkarnain Zainudin \\ Faculty of Electronics and Computer Engineering, \\ Universiti Teknikal Malaysia Melaka (UTeM), \\ Hang Tuah Jaya, 76100 Durian Tunggal, \\ Melaka, Malaysia \\ Email: zul@utem.edu.my
}

\author{
Sarath Kodagoda \\ Centre for Autonomous Systems (CAS), \\ Faculty of Engineering and Information Technology, \\ University of Technology Sydney, PO Box 123 Broadway, \\ Ultimo, NSW 2007, Australia \\ Email: Sarath.Kodagoda@uts.edu.au
}

\begin{abstract}
Generally, a model on describing human motion patterns should have an ability to enhance tracking performance particularly when dealing with long term occlusions. These patterns can be efficiently learned by applying Gaussian Processes (GPs). However, the GPs can become computationally expensive with increasing training data with time. Thus, with the proposed data selection and management using Mutual Information (MI) and Mahalanobis Distance (MD)approach, we have be able to keep the necessary portion of informative data and discard the others. This approach is then experimented by using the measurements of horizontal 2D scan of public area of our research centre with a stationary laser range finder. Experimental results show that even $90 \%$ reduction of data did not contribute to significantly increased Root Mean Square Error (RMSE). Implementation of Gaussian Process - Particle filter tracker for people tracking with long term occlusions produces a remarkable tracking performance when compared to Extended Kalman Filter (EKF) tracker.
\end{abstract}

\section{INTRODUCTION}

An ability to track people in the surroundings is a major interest in the fields of mobile service robot and human robot interaction in order to provide and build service robot attach to particular tasks and provides services to individual needs [1] [2]. In the past decade, various approaches and techniques have been introduced for people tracking in horizontal view scattered environment using multiple sensors like laser range finder and camera.

The implementation of state estimation using various tracking algorithms such as Kalman Filter(KF) [3], Particle Filter (PF) [4] and the interactive multiple model (IMM) [5] has improved tracking capabilities, however they are prone to errors when dealing with highly manoeuvering occluded targets. One way of handling such agile and occluded scenarios is to utilize a priory information such as target behaviours. The behaviours can be learned and utilized in the prediction stage. Inline with the statement, J. Ko et al [6] has introduced on learning of object motion patterns and incorporating the learned Gaussian Process regression models into various tracker.

Some researchers [7] [8], found that human motion has common patterns which depends on physical motion and environmental constraints to be embedded with tracking algorithms. GP learned models with a combination of extreme modelling flexibilities and consistent uncertainty estimation are then incorporated with probabilistic filtering techniques such as particle filter (PF) [9].

The increment of data has a long term data management problem which lead to computational intractability in the learning process of GPs such as in the work done by J. Ko et al [6]. It contributes to an accumulation of insignificant data because some training data may represent almost similar characteristics that leads to data management instability. Thus, we have introduced MI [10] and MD criteria [11] techniques to efficiently keep most informative data points.

This paper is arranged as follows. In section II, we present on how to manage and select data. Section III presents on details of Gaussian Process. Section IV presents the combination on Gaussian Process - Particle Filter. Section V explains experiment scenario. Section VI discusses experimental outcomes with multiple processes of optimized number of points. Section VI conclusion of the works.

\section{DATA SELECTION AND MANAGEMENT}

Normally, all the incoming data in GP will be periodically added to the training samples to accommodate new observations. In data selection and management process, all data has to gone through into three processing stages whenever the incoming observations are available. However, it is unnecessarily to include all new observations for the GP training data, the new observations need to be included only and if only they are informative. The motion models based on GP regression are timely adapted to accommodate data variations. This kind of issue can be solved by implementing a Mutual Information (MI) and Mahalanobis Distance (MD) based strategy to select and prioritize all data.

Initially, GP regression is represented by least number of informative data points that sequentially arranged by MI and satisfies the setup characteristics. Then, MD calculates value of each data points between the new measurement (NM) and the initial measurement (IM). Value of MD on data points that is lying within a decided value of the confidence interval will be discarded because the present GP is capable to represent the data. On the other hand, if it is more than the value of the confidence interval, the data needs to be included for the GP to represent the data. This process will iteratively continue when a new observation is available for training purposes and GP will adapt to the new scenarios. 


\section{A. Mutual Information}

Selection on the highly informational data points are based on Mutual Information (MI) algorithm [10]. Start with empty set of location $C=\phi$ and sequentially add points until $|C|=$ $t$ where $t$ is number of best points that initially appointed. Then, algorithm iteratively chooses the following points that give rise to the maximum increment on MI and ends when all $t$ data points are sequentially arranged. More particularly, the MI between the subset $A$ and the remaining points of trajectory $V \backslash C$ can be expressed as [10]:

$$
F(C)=I(C ; V \backslash C)
$$

Once $x \in V \backslash C$ is chosen and added to $C$, the variation of MI can be calculated by:

$$
\begin{array}{r}
F(C \cup x)-F(C)= \\
=H(C \cup x)-H(C \cup x \mid \bar{C})-[H(C)-H(C \mid \bar{C} \cup x)] \\
=H(x \mid C)-H(x \mid \bar{C})
\end{array}
$$

\section{B. Mahalanobis Distance}

The selection of new data that are going to incorporated together with the existing data in the GP learned model is decided by using Mahalanobis Distance (MD) [11]. With post MD analysis new data inclusion, adaptability of GP will be improved inline with the dynamically changing environments. Let assume that a successive measurement with value of variance $\sigma_{x m}$ and mean $\mu_{x m}$ was received at a location $\mathbf{x}_{i}$, where $\mathbf{x}=\langle x, y\rangle$. Thus, the prediction of variance $\sigma_{x p}$ and mean $\mu_{x p}$ can be carried out by using the new GP learned model.

The MD can be determined as

$$
d(\mathbf{x})=\sqrt{\frac{\left(\mu_{\mathbf{x} m}-\mu_{\mathbf{x} p}\right)^{2}}{\sigma_{\mathbf{x} m}^{2}+\sigma_{\mathbf{x} p}^{2}}} .
$$

The measurement threshold for $d(\mathbf{x})$ is referred to the chisquare table. It is chosen as 3.84 [12] and set to be within $95 \%$ confidence interval.

\section{GAUSSIAN PROCESS}

A Gaussian Process (GP) represents posterior distribution over function based on a set of training data where a natural generalization of linear regression of sample data with a collection of indiscriminate variables that allows us to consider uncertainty about predictions [13].

Let a set of training data, $D=\langle X, \mathbf{y}\rangle$, where $X=$ $\left[\mathbf{x}_{1}, \mathbf{x}_{2}, \ldots, \mathbf{x}_{n}\right]$ where it is a matrix representing d-dimensional input samples and $\mathbf{y}=\left[y_{1}, y_{2}, \ldots, y_{n}\right]$ is a vector representing scalar output. Gaussian Process (GP) regression output is modelled using a noisy version of function with the sampling data is derived from a noisy process,

$$
\mathbf{y}=f(\mathbf{x})+\varepsilon,
$$

where $\varepsilon$ is zero mean additive Gaussian noise with a variance of $\sigma_{n}^{2}$. With training data $D=\langle X, \mathbf{y}\rangle$ and a test input $\mathbf{x}_{*}$, a
GP defines a Gaussian predictive distribution over the output $\mathbf{y}_{*}$ with mean

$$
\mathrm{GP}_{\mu}\left(\mathbf{x}_{*}, D\right)=\mathbf{k}_{*}^{T}\left[K+\sigma_{n}^{2} I\right]^{-1} \mathbf{y}
$$

and variance

$$
\mathrm{GP}_{\Sigma}\left(\mathbf{x}_{*}, D\right)=k\left(\mathbf{x}_{*}, \mathbf{x}_{*}\right)-\mathbf{k}_{*}^{T}\left[K+\sigma_{n}^{2} I\right]^{-1} \mathbf{k}_{*}
$$

where, $\mathbf{k}_{*}$ is a vector defined by kernel values between the test input $\mathbf{x}_{*}$ and the training inputs $\mathbf{x} . K$ is the $n \times n$ kernel matrix of training input values $\mathbf{k}[m]=k\left(\mathbf{x}_{*}, \mathbf{x}_{m}\right)$ and $K[m, n]=k\left(\mathbf{x}_{m}, \mathbf{x}_{n}\right)$. The variance $\mathrm{GP}_{\Sigma}$, which uncertainty prediction that is depending on the process noise; and the correlation between the testing data and the training data. The squared exponential function which commonly used as a kernel function, is then selected for this process as given by,

$$
k\left(\mathbf{x}, \mathbf{x}^{\prime}\right)=\sigma_{f}^{2} e^{-\frac{1}{2}\left(\mathbf{x}-\mathbf{x}^{\prime}\right) W\left(\mathbf{x}-\mathbf{x}^{\prime}\right)^{T}}
$$

where $\sigma_{f}^{2}$ is the signal variance. $W$ is each input dimension with the length scales for in a diagonal matrix .

In Gaussian Process, the kernel function and the process noise are known as hyperparameters. They are learned using numerical optimization techniques such conjugate gradient decent [13] on maximization the log-likelihood of training data. Let assume a d-dimensional track $V$ has $|V|$ total number of data points. Based on GP regression models, we can predict value at any point $y \in V \backslash A$ if we observe a set of points, $A \subset V$. Let signify $z_{y}$ as a value at $y$ and $Z_{A}$ as a set of values at the finite set $A$. Derivation of conditional distribution at predicted point of $y$ at given $Z_{A}$ in probabilistic terms is shown as follows [6]:

$$
\begin{gathered}
\mu_{y \mid A}=\mu_{y}+\Sigma_{y A} \Sigma_{A A}^{-1}\left(Z_{A}-\mu_{A}\right) \\
\sigma_{y \mid A}^{2}=k(y, y)-\Sigma_{y A} \Sigma_{A A}^{-1} \Sigma_{A y}
\end{gathered}
$$

where $\Sigma_{y A}$ is a one input covariance vector for each $\mathbf{x} \in A$ with value $k(y, \mathbf{x}) ; \mu_{y \mid A}$ and $\sigma_{y \mid A}^{2}$ are conditional mean and variance at $\mathrm{y} ; \mu_{A}$ is a mean vector of $Z_{A}$; and $\Sigma_{A A}^{-1}$ is a covariance matrix of $Z_{A}$ with each input calculated by $k(\mathbf{x}, \mathbf{x})$.

\section{GAUSSIAN PROCESS - PARTICLE FILTER}

Particle Filter (PF) is a Bayesian estimator that resolve problems for nonlinear prediction in the dynamics and measurements by determining the probabilistic density function (PDF) of unknown random vector using a weighted sum of delta functions. Particle Filter with the problems on learning prediction and measurement can be determine with the assistance of Gaussian Process regression. In this work, a static observer was chosen to train prediction models in order to ensure the validity of this technique. The state and control $\left(\mathbf{x}_{k}, \mathbf{u}_{k}\right)$ is then mapped to the state transition, $\Delta \mathbf{x}_{k}=$ $\mathbf{x}_{k+1}-\mathbf{x}_{k}$. An addition of the state transitions to the previous state yields the following state of process model. Training set of data on prediction and observation is represented by 


$$
D_{p}=\left\langle(X, U), X^{\prime}\right\rangle
$$

where $X$ is location coordinates matrix and $X^{\prime}=$ $\left[\Delta x_{1}, \Delta x_{2}, \ldots, \Delta x_{k}\right]$ is transitional matrix made from those states when applying the controls stored in $U$.

$$
\begin{aligned}
& p\left(\mathbf{x}_{k} \mid \mathbf{x}_{k-1}, \mathbf{u}_{k-1}\right) \\
& \quad \approx N\left(\operatorname{GP}_{\mu}\left(\left[x_{k-1}, \mathbf{u}_{k-1}\right], D_{p}\right), \operatorname{GP}_{\Sigma}\left(\left[x_{k-1}, \mathbf{u}_{k-1}\right], D_{p}\right)\right)
\end{aligned}
$$

The main task of particle filter is to represent posteriors over the state $\mathbf{x}_{k}$ by setting $X_{k}$ of weighted samples:

$$
X_{k}=\left\{\left\langle\mathbf{x}_{k}^{m}, w_{k}^{(m)}\right\rangle \mid m=1, \ldots, M\right\} .
$$

Here, each $\mathbf{x}_{k}^{m}$ is a sample and each $w_{k}^{(m)}$ is a non-negative numerical factor called importance weight. This term, $\operatorname{GP}\left(\left[x_{k-1}, \mathbf{u}_{k-1}\right], D_{p}\right)$ is the short form of the Gaussian represented by $\left(\mathrm{GP}_{\mu}\left(\left[x_{k-1}, \mathbf{u}_{k-1}\right], D_{p}\right), \mathrm{GP}_{\Sigma}\left(\left[x_{k-1}, \mathbf{u}_{k-1}\right], D_{p}\right)\right)$.

Generally, local density of training data will give different covariance values on the prediction for each sample. The details on flow of the process can be referred in [6].

\section{EXPERIMENT SCENARIO}

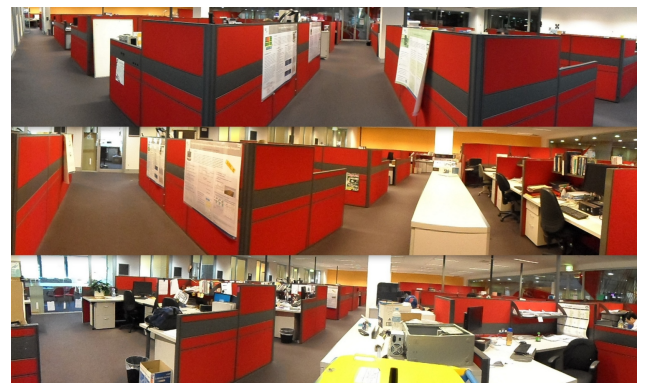

Fig. 1. Horizontal view of the Centre of Autonomous Systems

Experimental data for the proposed analyses were carried out and collected in a public area of our research centre as it can be horizontally viewed in Fig. 1. In order the data to be properly collected, the laser range finder is set in a stationary position to horizontally view and scan the environment. The laser range finder used is HOKUYO UTM-30LX laser range finder with capability of 30 meters detection range, 25 millisecond sampling period, $270^{\circ}$ angular field of view and $0.25^{\circ}$ angular resolution. LRF is fitted with onboard computer that is running on Linux Ubuntu operating system.

In the experiment, a person that subjected as a target has walked multiple times at four designated trajectories for the LRF to observe and scan the target. The LRF vertical position is set at a torso height of adult and slightly above the partition to detect the target. The detection process consists of extraction on designated features assisted by a classification process using learning algorithm [14]. The target is then represented by cartesian coordinates that is converted from polar coordinates and later used for the GP modelling. Fig. 2 shows all trajectories with 10 paths in one direction.
Finally, the GP-PF is then implemented as a tool for tracking the target with setup average root mean square error (ARMSE) on the prediction within $5 \mathrm{~cm}$ as the width of walking paths between 130 and $150 \mathrm{~cm}$.

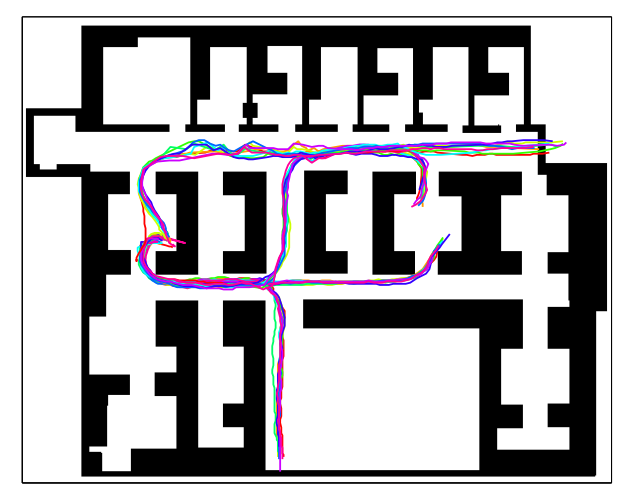

Fig. 2. Trajectories of a walking person on a simple map of the common area

\section{RESULTS}

The training data has been experimentally analysed in three processes. MI analysis is used to determine the least number of data points representing the GP distribution within the ARMSE. Then, MD analysis will ensure whether each new observation has contribute to the new knowledge. Again, MI analysis will remove any unnecessary data points from the set of new data. The GP model with covariance and mean values in 3D plot are shown in Fig. 4 and Fig. 5, respectively.

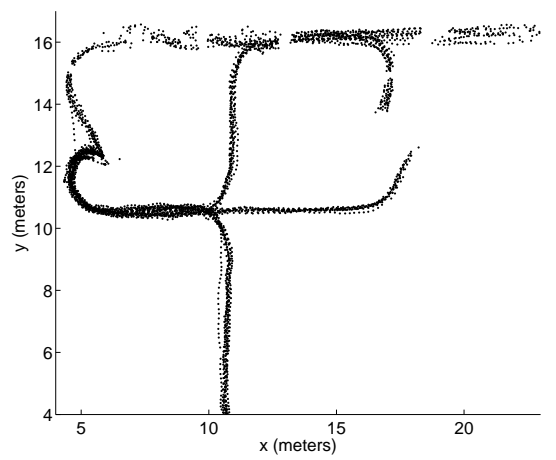

Fig. 3. 3868 dots represents trajectories of the subject

The figure shown in Fig. 3 shows all the four designated routes with 10 trajectories on each one. It contains 3868 points with the point of observation is coordinate $(10,10)$. MD analysis on predicted and measured values of mean and covariance is carried out whenever new observation was captured to each point in $\mathrm{x}$ and $\mathrm{y}$ axes. MD analysis will then discard all the points that are below the threshold 3.84 (refer to any $\chi^{2}$ table). For instance, all the observation points that have more MD values that 3.84 are no longer representing the GP regression values as shown in Fig. 6 and Fig. 7 will be added to the set of data points. 
Less MDs means the observations are assumed belonging to the GP model and hence they do not add any extra information to the current model. The data points that are higher than the threshold will then be incorporated to the training samples for the next MI process as shown in Fig. 6 and Fig. 7. The post MD analysis data is iteratively chosen using MI analysis until RMSE of set of data points within 5 $\mathrm{cm}$.

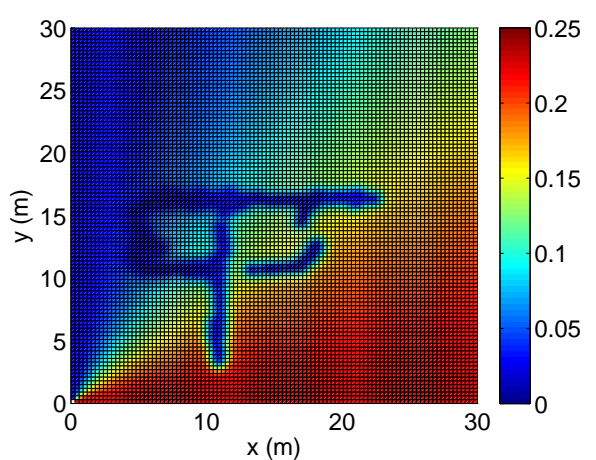

Fig. 4. Covariance Values before applying MI and MD

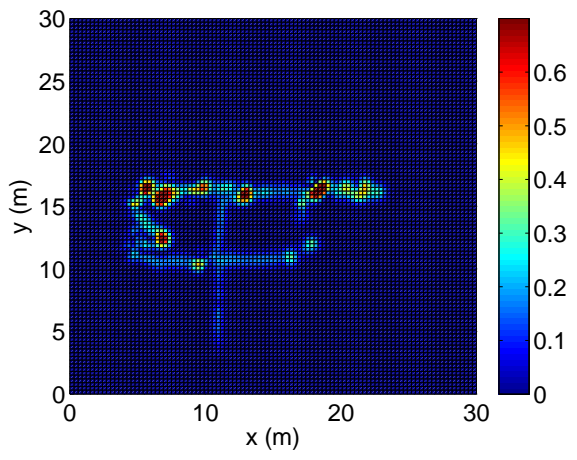

Fig. 5. Mean Values before applying MI and MD

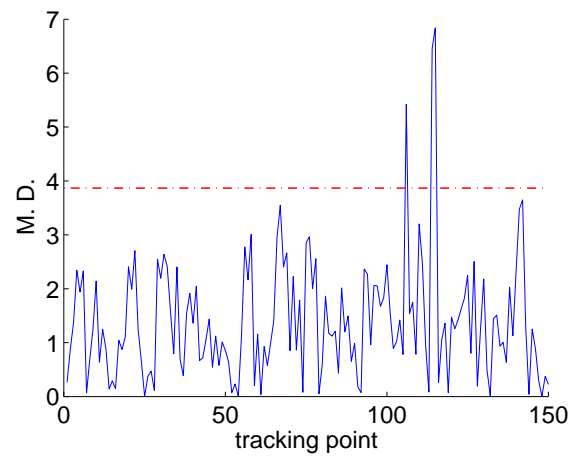

Fig. 6. Mahalanobis Distance values in the $\mathrm{x}$ direction

The chosen data based on MD analysis is then selected by MI analysis for most informative data points selection as shown in Fig. 8. Selected data have the RMSE less than 0.02 meter with ARMSE is 0.0225 meter as shown in Fig. 9.

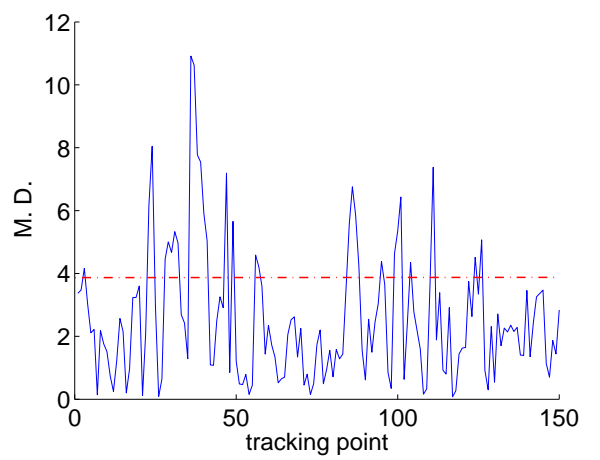

Fig. 7. Mahalanobis Distance values in the y direction

The results shown in Fig. 11 and Fig. 10 are the mean and covariance after GP training. It is clearly seen that the uncertain values of GP predictions are high when there is no observations.

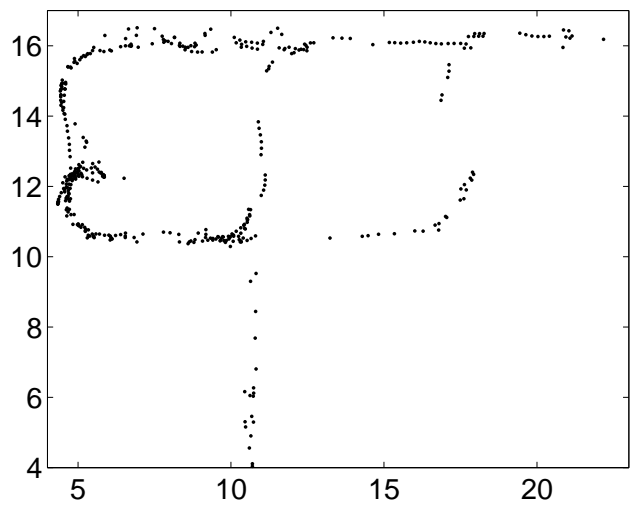

Fig. 8. 363 points in four routes after applying MI and MD

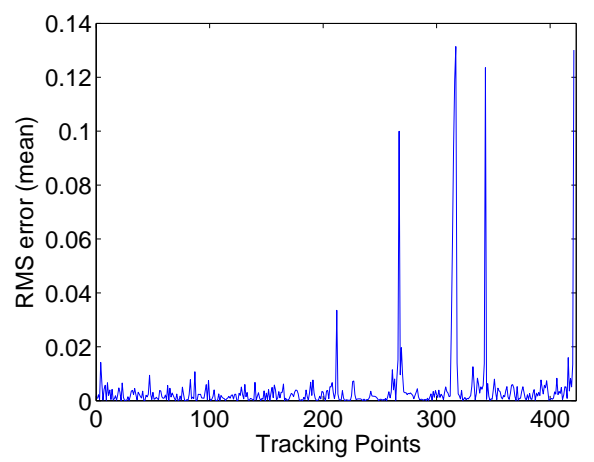

Fig. 9. RMS Error of the Predicted Mean

Referring to Fig. 3 and Fig. 8, the total number of informative data that is used for training was reduced from 3868 points to 363 points, as a result achieving more than a $90 \%$ reduction of data.

Fig. 12 refers to people tracked on 4 routes simultaneously without any occlusion. Green line represents people tracked 


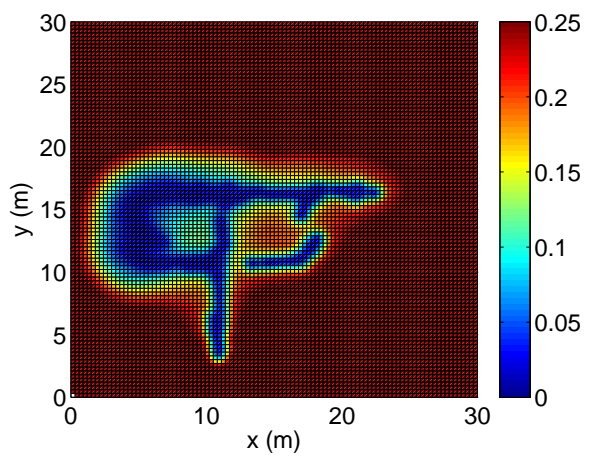

Fig. 10. Covariance Values after applying MI and MD

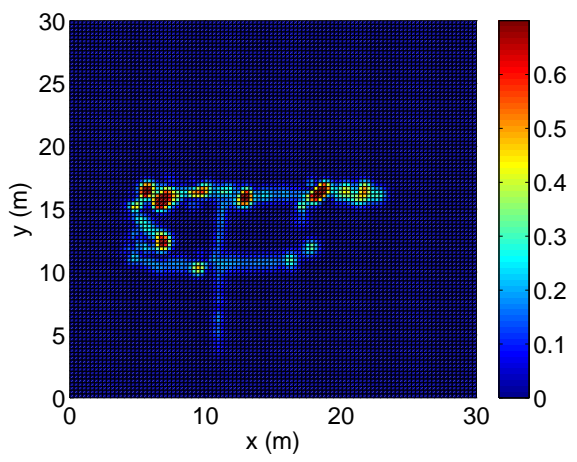

Fig. 11. Mean Values after applying MI and MD

by GP-PF, red line represents people tracked by EKF, black dots are reference points or ground truth and blue dots are the particle of GP-PF. The GP=PF tracking performance is better than EKF referring to ground truths. This GP model is then embedded with PF tracker for the analysis on the long term tracking ability. The results of analysis on both models for 1 route with an occlusion is shown in Fig. 13. The occlusion occurs from point $(11,12.6)$ and ends at point $(17.5,16.1)$. GP-PF tracker out-performed EKF tracker where

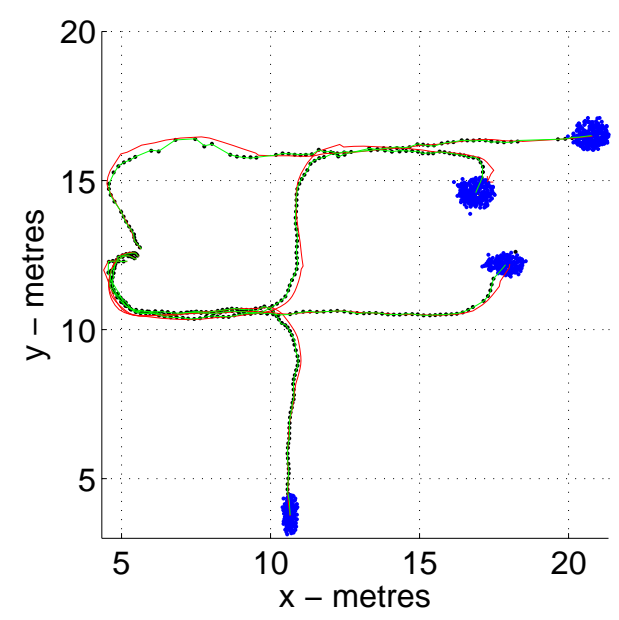

Fig. 12. Tracking results for 4 routes without occlusion: GP-PF (green), EKF (red)and black dots (ground truth).

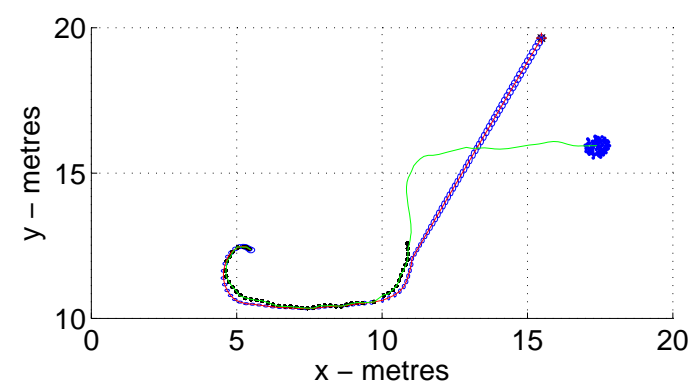

Fig. 13. Tracking on 1 route with partial occlusion by using GP-PF (green) and EKF (red).

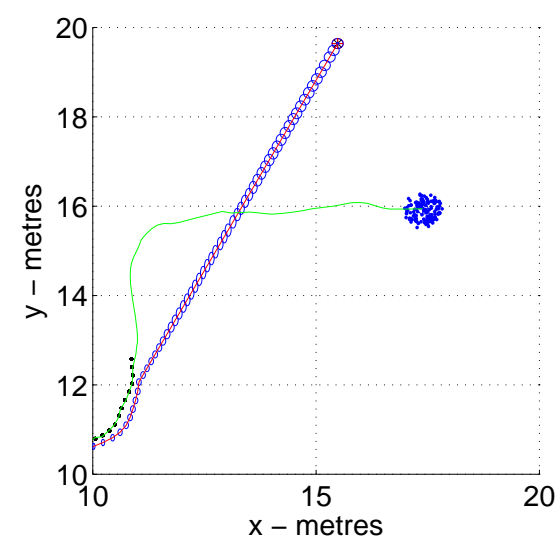

Fig. 14. Zoom in on growing covariance ellipses of occlusion period by EKF tracker.

the GP-PF managed to continue prediction with growing covariance while EKF tracker lost track. The covariance ellipse continues growing with prediction as appear on Fig. 14.

Fig. 15 shows the simultaneous tracking on 2 routes with partial occlusion on both routes by EKF tracker and GP-PF tracker. Again the EKF tracker has shown extremely poor tracking accuracies on both routes, contrarily the GP-PF tracker shows superior tracking performance on the occlusions with certain period of time.

Fig. 16 and Fig. 17 show the simultaneous tracking on 3 routes and 4 routes with partial occlusions, respectively. Both figures show the superior tracking performance of GPPF tracker.

\section{CONCLUSIONS}

It is noted that the implementation of method for discarding least informative data is successfully carried out. $90 \%$ of data points reduction contribute to computational savings in particular when periodically dealing with large volume of data. GP model for simultaneously tracking people in complex indoor environment is then incorporated in PF to track people in the multiple routes. Data reduction using this technique contributes to computational savings since GP computation for the large amount of data is computationally expensive. The learned GP was then incorporated in the $\mathrm{PF}$ for tracking people in the various routes. From the 


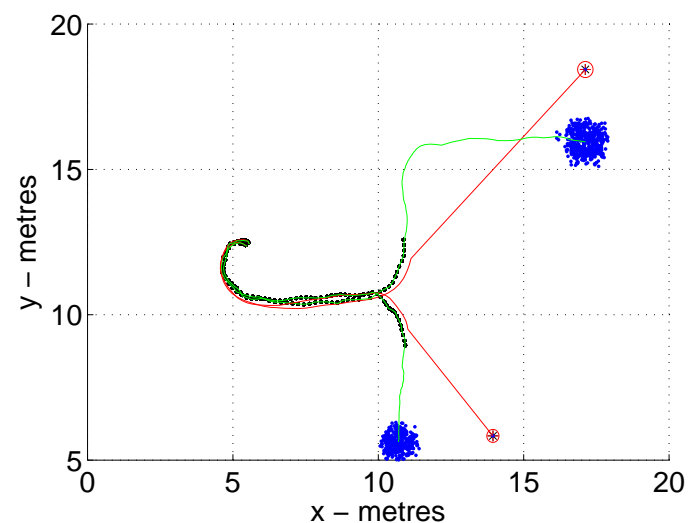

Fig. 15. Tracking on 2 routes with partial occlusion by using GP-PF (green) and EKF (red).

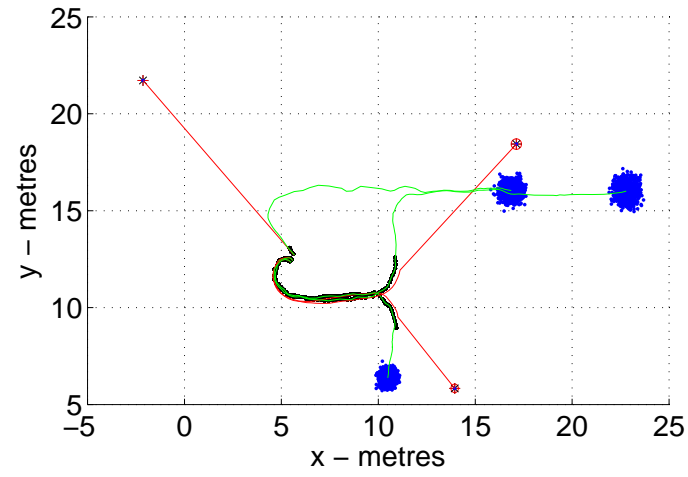

Fig. 16. Tracking on 3 routes with partial occlusion by using GP-PF (green) and EKF (red).

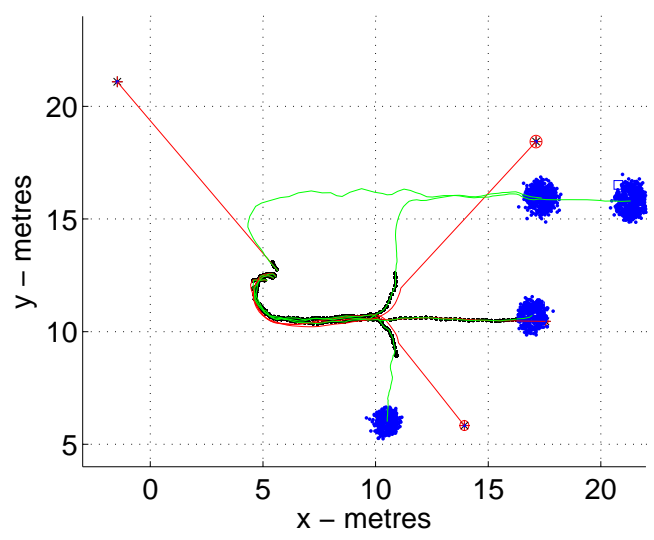

Fig. 17. Tracking on 3 routes with partial occlusion by using GP-PF (green) and EKF (red). experiments, GP-PF is proven to produce superior tracking performance with occlusions when comparing with EKF tracker.

In our future work, we intend to combine this algorithm with other techniques on various complex scenarios.

\section{ACKNOWLEDGMENT}

This work is jointly supported by Faculty of Electronic and Computer Engineering, Universiti Teknikal Malaysia Melaka (UTeM), Malaysia, the Centre of Autonomous Systems (CAS), University of Technology, Sydney, Australia and the Ministry of Higher Education (MoHE), Malaysia.

\section{REFERENCES}

[1] L. K. Geoffrey Taylor, "A multiple hypothesis walking person tracker with switched dynamic model," 2004

[2] D. F. Glas, T. Miyashita, H. Ishiguro, and N. Hagita, "Laser-based tracking of human position and orientation using parametric shape modeling," Advanced robotics, vol. 23, no. 4, pp. 405-428, 2009.

[3] D. Schulz, W. Burgard, D. Fox, and A. B. Cremers, "People tracking with mobile robots using sample-based joint probabilistic data association filters," The International Journal of Robotics Research, vol. 22, no. 2, pp. 99-116, 2003.

[4] D. Schulz, D. Fox, and J. Hightower, "People tracking with anonymous and id-sensors using rao-blackwellised particle filters," in IJCAI, 2003, pp. 921-928.

[5] K. R. S. Kodagoda, S. S. Ge, W. S. Wijesoma, and A. P. Balasuriya, "Immpdaf approach for road-boundary tracking," vol. 56, no. 2, pp. 478-486, 2007. [Online]. Available: http://ieeexplore.ieee.org/stamp/ stamp.jsp?arnumber $=4138030$

[6] J. Ko and D. Fox, "Gp-bayesfilters: Bayesian filtering using gaussian process prediction and observation models," Autonomous Robots, vol. 27, no. 1, pp. 75-90, 2009. [Online]. Available: http://www.springerlink.com/index/PX45033851UP28R5.pdf

[7] S. Morishita, A. Nishimura, and H. Asama, "A method to estimate destination of a walking person with hidden markov model for safety of human friendly robots," in Safety, Security and Rescue Robotics, 2008. SSRR 2008. IEEE International Workshop on. IEEE, 2008, pp. 115-120. [Online]. Available: http://ieeexplore.ieee.org/xpls/abs_all.jsp?arnumber=4745887

[8] A. Escobedo, A. Spalanzani, C. Laugier, et al., "Experimental setup for human aware navigation," in Control Architectures of Robots, 2012. [Online]. Available: http://hal.inria.fr/hal-00746567/

[9] C. Plagemann, D. Fox, and W. Burgard, "Efficient failure detection on mobile robots using particle filters with gaussian process proposals," in Proc. of the Twentieth International Joint Conference on Artificial Intelligence (IJCAI), 2007. [Online]. Available: http: //www.aaai.org/Papers/IJCAI/2007/IJCAI07-352.pdf

[10] C. Guestrin, A. Krause, and A. Singh, "Near-optimal sensor placements in gaussian processes," in Proceedings of the 22nd international conference on Machine learning. ACM, 2005, pp. 265-272. [Online]. Available: http://dl.acm.org/citation.cfm?id=1102385

[11] R. De Maesschalck, D. Jouan-Rimbaud, and D. L. Massart, "The mahalanobis distance," Chemometrics and Intelligent Laboratory Systems, vol. 50, no. 1, pp. 1-18, 2000. [Online]. Available: http://www.sciencedirect.com/science/article/pii/S0169743999000477

[12] G. Schay, Introduction to probability with statistical applications. Birkhäuser, 2007.

[13] C. Rasmussen and C. Williams, Gaussian processes for machine learning. MIT press Cambridge, MA, 2006, vol. 1.

[14] Z. Zainudin, S. Kodagoda, and G. Dissanayake, "Torso detection and tracking using a 2d laser range finder," in Proceedings of the Australasian Conference on Robotics and Automation, 2010. 